\title{
Temporal changes in the biomass and distribution of Sargassum beds along the southeastern coast of the Baja California Peninsula
}

\section{Cambios temporales de la biomasa y distribución de los mantos de Sargassum en la costa sureste de la península de Baja California}

\author{
Margarita Casas-Valdez ${ }^{1 *}$, Ignacio Sánchez-Rodríguez ${ }^{1 \dagger}$, Elisa Serviere-Zaragoza² ${ }^{2}$ Ruth N Aguila-Ramírez ${ }^{1}$ \\ ${ }^{1}$ Centro Interdisciplinario de Ciencias Marinas, Instituto Politécnico Nacional (CICIMAR-IPN), Avenida \\ Instituto Politécnico Nacional s/n, La Paz 23096, Baja California Sur, México. \\ 2 Centro de Investigaciones Biológicas del Noroeste (CIBNOR), Avenida Instituto Politécnico Nacional \#195, \\ La Paz 23096, Baja California Sur, México. \\ * Corresponding author. E-mail: mcasasv@hotmail.com
}

\begin{abstract}
Changes in the distribution, surface canopy, biomass, and standing crop of Sargassum beds (Phaeophyceae) along the southeastern coast of the Baja California Peninsula (Mexico) are described and compared with previous estimates. Also, Sargassum biomass was correlated with latitude. Species were identified and surface canopy, biomass, standing crop, and length structure were estimated for 72 Sargassum beds in 3 localities in 2006. The extent of each bed was determined using GPS. The perimeter of each bed was drawn and its canopy area was calculated. The average biomass was estimated, using quadrats that were randomly distributed over the beds. The highest average biomass was found at Mulegé, with significant differences in the other 2 study areas (Concepción and La Paz bays). The largest bed, surface canopy area, and standing crop were recorded in Concepción Bay. Coverage area, location, and seasonal changes in surface canopy, standing crop, and biomass of Sargassum beds along the southeastern coast of the Baja California Peninsula were compared with the different phenological stages of Sargassum thalli, variations in the available rocky sea floor, and oceanographic characteristics. These aspects must be considered to maintain sustainable exploitation of this potential resource.
\end{abstract}

Key words: biomass, Gulf of California, Sargassum, standing crop, seasonal changes.

RESUMEN. Se describen y comparan con estimaciones previas los cambios en la distribución, superficie, biomasa y cosecha de mantos de Sargassum (Phaeophyceae) a lo largo de la costa sureste de la península de Baja California (México). También, la biomasa de Sargassum se correlacionó con la latitud. Se determinaron las especies y se estimó la superficie, biomasa, cosecha y estructura de tallas de 72 mantos de Sargassum en 3 zonas en 2006. La extensión de cada manto fue determinada usando GPS. El perímetro de cada manto fue dibujado y su superficie fue calculada. La biomasa promedio fue estimada usando cuadrantes que fueron distribuidos al azar sobre los mantos. Se registró la biomasa promedio más alta en Mulegé, encontrándose diferencias significativas con respecto a las otras 2 zonas de estudio (bahías Concepción y La Paz). En bahía Concepción se registró el manto más grande y la mayor superficie y cosecha. Los cambios en la distribución, superficie, biomasa y cosecha de los mantos de Sargassum a lo largo de la costa sureste de la península de Baja California fueron comparados con diferentes estadios fenológicos de Sargassum, variaciones en la disponibilidad de sustrato rocoso y características oceanográficas. Estos aspectos deben ser considerados para mantener una explotación sustentable de este recurso potencial.

Palabras clave: biomasa, golfo de California, Sargassum, cosecha, cambios estacionales.

\section{INTRODUCTION}

The genus Sargassum C Agardh (Phaeophyceae, Fucales, Sargassaceae) has a worldwide distribution, especially in tropical and subtropical regions (Noiraksar et al. 2014), including the Gulf of California (Pacheco-Ruíz et al. 1998). In this gulf, Sargassum species constitute the bulk of the fleshy macroalgal biomass, providing habitat for diverse communities of epiphytic algae and a surface canopy for understory algae, invertebrates, and fish (Norris 2010).

Sargassum species from the Gulf of California could serve as a source of alginate (Rodríguez-Montesinos et al. 2008) and could be used in agricultural fertilizers, in forage for goats and sheep, in balanced diets for chickens and

\section{INTRODUCCIÓN}

El género Sargassum C Agardh (Phaeophyceae, Fucales, Sargassaceae) presenta una amplia distribución, especialmente en regiones tropicales y subtropicales (Noiraksar et al. 2014), incluyendo el golfo de California (Pacheco-Ruíz et al. 1998). En el golfo, las especies de Sargassum constituyen la mayoría de la biomasa de macroalgas carnosas, y proporcionan hábitat para diversas comunidades de algas epifíticas y una cubierta para algas, invertebrados y peces del sotobosque (Norris 2010).

Las especies de Sargassum del golfo de California podrían servir como fuente de alginato (RodríguezMontesinos et al. 2008) y podrían usarse en fertilizantes para 
shrimp, and in human nutrition (Marín-Álvarez et al. 2003; Casas-Valdez et al. 2006a, 2006b; Casas-Valdez 2009). Extracts of Sargassum have antimicrobial activity against pathogenic organisms, and hence, possess potential in the pharmaceutical industry (Muñoz-Ochoa et al. 2010). Studies of Sargassum sinicola determined some potential uses of alginate as raw material for cell immobilization in wastewater treatment and promoting plant growth (Yabur et al. 2007), and capacity to absorb heavy metal wastes from mining industries (Huerta-Díaz et al. 2007, Patrón-Prado et al. 2010). To date, Sargassum beds have not been harvested in the Gulf of California for commercial purposes (Casas-Valdez 2009). For commercial planning purposes, it is necessary to estimate biomass (Andrefouet et al. 2004).

Research on Sargassum in the Gulf of California indicates a seasonal pattern of growth and abundance. In general, the maximum growth rate and length of the thallus occur in the spring, as reported for S. sinicola (Núñez-López and CasasValdez 1997) and S. lapazeanum (Rivera and Scrosati 2006). In spring, the highest growth rates coincide with the maximum biomass reported for species along the eastern coast of the Baja California Peninsula: S. sinicola (Espinoza-Avalos 1990), S. horridum (Muñeton and Hernández-Carmona 1993), and S. lapazeanum (Rivera and Scrosati 2006) in La Paz Bay; S. sinicola, S. herporhizum, and S. lapazeanum (Núñez-López and Casas-Valdez 1996) in Concepción Bay; and S. herporhizum, S. johnstonii, and S. sinicola from Mulegé to San Luis Gonzaga (Pacheco-Ruíz et al. 1998). Sargassum has maximum growth, length of thallus, and biomass when the water temperature is relatively cold, which occurs in spring, and the slowest growth during late summer (Espinoza-Avalos 1993).

The total biomass of Sargassum beds (in wet tons) has been estimated at several sites along the eastern coast of the peninsula: La Paz Bay $(18,900 \pm 928)$, Concepción Bay $(7,250 \pm$ 530), and from Mulegé to San Luis Gonzaga (154,559 \pm 16,139) (Hernández-Carmona et al. 1990, Casas-Valdez et al. 1993, Pacheco-Ruíz et al. 1998). However, to the best of our knowledge, these are the last estimates to have been published and current estimates are necessary because the distribution and coverage may have changed in the past 2 decades.

To update the information, the location, surface canopy area, biomass, and standing crop of Sargassum beds along the southeastern coast of the Baja California Peninsula (Mexico) were surveyed and compared with previous studies. We also determined whether biomass was related to latitude. To assess the potential for sustainable exploitation, a new database of information on coverage and seasonal abundance for Sargassum, within this area, is vital.

\section{MATERIALS AND METHODS}

\section{Study area}

Surveys were made from La Ventana (southern La Paz Bay; $\left.24^{\circ} 02^{\prime} \mathrm{N}, 109^{\circ} 59^{\prime} \mathrm{W}\right)$ to La Salina $\left(27^{\circ} 52^{\prime} \mathrm{N}, 112^{\circ} 46^{\prime} \mathrm{W}\right)$ la agricultura, en forraje para cabras y ovejas, en dietas balanceadas para pollos y camarones y en la alimentación humana (Marín-Álvarez et al. 2003; Casas-Valdez et al. 2006a, 2006b; Casas-Valdez 2009). Los extractos de Sargassum presentan actividad antimicrobiana contra organismos patógenos, por lo que tienen potencial para la industria farmacéutica (Muñoz-Ochoa et al. 2010). Estudios realizados con Sargassum sinicola han identificado algunos usos potenciales de alginato como materia prima para la inmovilización de células en el tratamiento de aguas residuales y para promover el crecimiento de plantas (Yabur et al. 2007), así como su capacidad para absorber residuos de metales pesados de la industria minera (Huerta-Díaz et al. 2007, Patrón-Prado et al. 2010). A la fecha, los mantos de Sargassum no han sido cosechados en el golfo de California para propósitos comerciales (Casas-Valdez 2009). Para poder realizar una correcta planificación comercial, es necesario estimar la biomasa (Andrefouet et al. 2004).

Las investigaciones sobre Sargassum en el golfo de California indican un patrón estacional de crecimiento y abundancia. En general, la máxima tasa de crecimiento y la máxima longitud del talo se registran en primavera, como ha sido documentado para S. sinicola (Núñez-López y CasasValdez 1997) y S. lapazeanum (Rivera y Scrosati 2006). En primavera, las tasas de crecimiento más altas coinciden con la máxima biomasa registrada para las especies en la costa este de la península de Baja California: S. sinicola (EspinozaAvalos 1990), S. horridum (Muñeton y Hernández-Carmona 1993) y S. lapazeanum (Rivera y Scrosati 2006) en bahía de La Paz; S. sinicola, S. herporhizum y S. lapazeanum (NúñezLópez y Casas-Valdez 1996) en bahía Concepción; y S. herporhizum, S. johnstonii y S. sinicola de Mulegé a San Luis Gonzaga (Pacheco-Ruíz et al. 1998). El crecimiento, la longitud del talo y la biomasa de Sargassum son mayores cuando la temperatura del agua es relativamente fría, lo que sucede en primavera, y el crecimiento es menor a finales de verano (Espinoza-Avalos 1993).

Se ha estimado la biomasa total de los mantos de Sargassum (en toneladas húmedas) en varios sitios a lo largo de la costa este de la península: bahía de La Paz $(18,900 \pm$ 928), bahía Concepción $(7,250 \pm 530)$ y de Mulegé a San Luis Gonzaga $(154,559 \pm 16,139)$ (Hernández-Carmona et al. 1990, Casas-Valdez et al. 1993, Pacheco-Ruíz et al. 1998); sin embargo, según nuestro conocimiento, éstas son las últimas estimaciones que se han publicado y se necesitan estimaciones actuales ya que la distribución y cobertura podrían haber cambiado en las últimas 2 décadas.

Para actualizar la información, se estudiaron y compararon con estudios previos la ubicación, área superficial, biomasa y cosecha de mantos de Sargassum en la costa sureste de la península de Baja California (México). También se examinó si había una relación entre la biomasa y la latitud. Para evaluar el potencial para una cosecha sustentable de Sargassum, es de suma importancia contar con una nueva base de datos de su cobertura y abundancia estacional en esta región. 
(Fig. 1). Three localities were studied: (I) Mulegé $\left(26^{\circ} 56^{\prime} \mathrm{N}\right.$, $111^{\circ} 58^{\prime} \mathrm{W}$ to $27^{\circ} 59^{\prime} \mathrm{N}, 112^{\circ} 43^{\prime} \mathrm{W}$ ), (II) Concepción Bay $\left(26^{\circ} 52^{\prime} \mathrm{N}, 111^{\circ} 54^{\prime} \mathrm{W}\right.$ to $\left.26^{\circ} 35^{\prime} \mathrm{N}, 111^{\circ} 43 \mathrm{~W}\right)$, and (III) La Paz Bay $\left(24^{\circ} 45^{\prime} \mathrm{N}, 110^{\circ} 28^{\prime} \mathrm{W}\right.$ to $\left.24^{\circ} 08^{\prime} \mathrm{N}, 110^{\circ} 24^{\prime} \mathrm{W}\right)$. These sites were selected because data of Sargassum beds were available for them. The specific location of each bed within each zone was the same or at least very similar to the sites of previous studies. This made comparisons more useful. The surveys were made during spring because the previous studies of Sargassum beds were performed during spring.

Along the coast of the Baja California Peninsula, there are conspicuous areas where Sargassum grows, each separated from its neighbor. These beds contain a community of species, where each community can grow up to $25 \mathrm{~m}$. The upper fronds reach the surface, forming a dense surface canopy. Our survey included 23 beds in zone I, 22 beds in zone II, and 27 beds in zone III.

\section{Proportion of species and length}

Since several species of Sargassum are found along this coast, the proportion of each species was determined by using a random sampling design in each bed. Three quadrats were distributed randomly. All individuals within a quadrate were collected and later identified to species, using 2 identification guides (Setchell and Gardner 1924, Dawson 1944). The Sargassum specimens were measured for length; 50 thalli of each species were measured from holdfast to

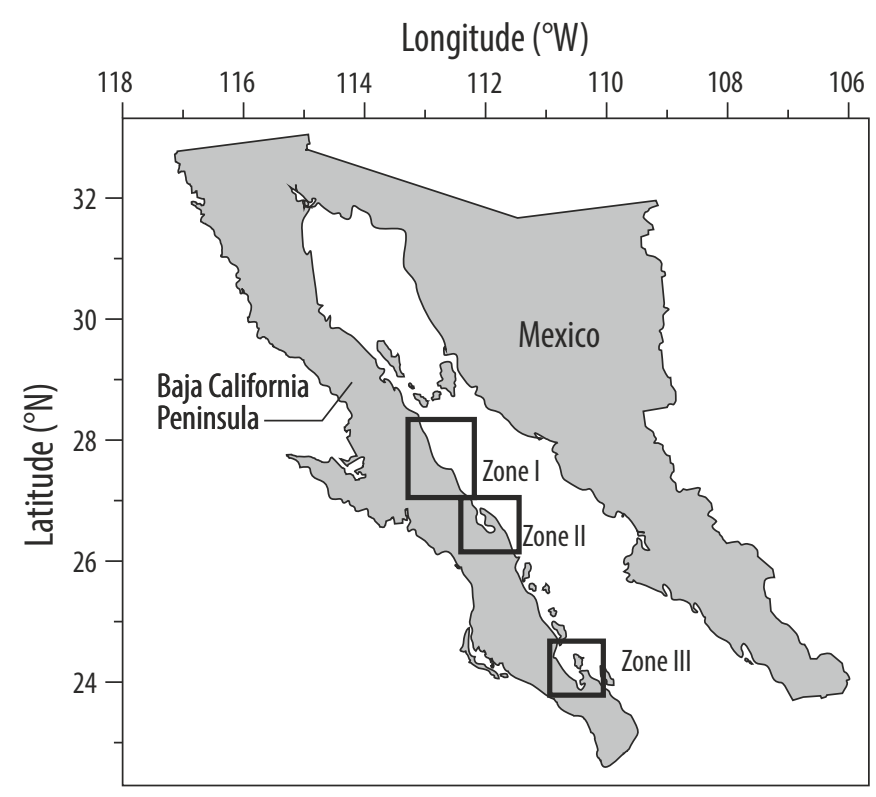

Figure 1. Study area, southeastern coast of the Baja California Peninsula (Mexico): Mulegé (zone I), Concepción Bay (zone II), and La Paz Bay (zone III).

Figura 1. Área de estudio, costa sureste de la península de Baja California (México): Mulegé (zona I), bahía Concepción (zona II) y bahía de La Paz (zona III).

\section{MATERIALES Y MÉTODOS}

\section{Área de estudio}

Los muestreos se realizaron entre La Ventana (sur de la bahía de La Paz; $\left.24^{\circ} 02^{\prime} \mathrm{N}, 109^{\circ} 59^{\prime} \mathrm{W}\right)$ y La Salina $\left(27^{\circ} 52^{\prime} \mathrm{N}\right.$, $112^{\circ} 46^{\prime} \mathrm{W}$ ) (Fig. 1). Se estudiaron 3 zonas: (I) Mulegé $\left(26^{\circ} 56^{\prime} \mathrm{N}, 111^{\circ} 58^{\prime} \mathrm{W}\right.$ a $\left.27^{\circ} 59^{\prime} \mathrm{N}, 112^{\circ} 43^{\prime} \mathrm{W}\right)$, (II) bahía Concepción $\left(26^{\circ} 52^{\prime} \mathrm{N}, 111^{\circ} 54^{\prime} \mathrm{W}\right.$ a $\left.26^{\circ} 35^{\prime} \mathrm{N}, 111^{\circ} 43 \mathrm{~W}\right)$ y (III) bahía de $\operatorname{La~} \operatorname{Paz}\left(24^{\circ} 45^{\prime} \mathrm{N}, 110^{\circ} 28^{\prime} \mathrm{W}\right.$ a $\left.24^{\circ} 08^{\prime} \mathrm{N}, 110^{\circ} 24^{\prime} \mathrm{W}\right)$. Estos sitios fueron seleccionados por contar con datos de los mantos de Sargassum. Para que las comparaciones fueran más útiles, la ubicación específica de cada manto dentro de cada zona fue igual o muy similar a los sitios de estudios previos. Los muestreos se realizaron en primavera ya que los estudios previos también fueron realizados en primavera.

A lo largo de la costa de la península de Baja California, hay zonas conspícuas donde crecen los mantos de Sargassum, cada una aislada de la zona vecina. Estos mantos albergan una comunidad de especies, donde cada comunidad puede crecer hasta $25 \mathrm{~m}$. Las hojas superiores llegan a la superficie y forman una densa bóveda arbórea. Nuestro estudio incluyó 23 mantos en la zona I, 22 mantos en la zona II y 27 mantos en la zona III.

\section{Proporción de especies y longitud}

A lo largo de esta costa se encuentran varias especies de Sargassum. La proporción de cada especie se determinó mediante un muestreo aleatorio en cada manto. Se distribuyeron 3 cuadrantes al azar. Todos los individuos dentro de un cuadrante fueron recolectados y subsecuentemente identificados a nivel de especie con base en 2 guías de identificación (Setchell y Gardner 1924, Dawson 1944). Se midió la longitud de los especímenes de Sargassum; 50 talos de cada especie fueron medidos desde el órgano de fijación hasta el ápice para obtener la longitud promedio en las 3 zonas. La longitud está directamente relacionada con la biomasa, como lo describen Rivera y Scrosati (2006).

\section{Área superficial, biomasa y cosecha}

Los mantos de Sargassum fueron clasificados como pequeños (1 a $500 \mathrm{~m})$, medianos (501 a 1,500 m) o grandes $(>1,501 \mathrm{~m})$ según su longitud (Hernández-Carmona et al. 1990, Casas-Valdez et al. 1993). Los mantos de Sargassum yacen paralelos a la línea de costa. La longitud del manto es la distancia lineal de cada manto paralelo a la costa, y la anchura es la distancia lineal desde la parte más cercana a la costa hasta la parte más lejana de la costa, perpendicular a la línea de costa. Para no sobrepasar un nivel de error del 10\%, se midió la anchura de los mantos cada $50 \mathrm{~m}$ en el caso de los pequeños, cada $100 \mathrm{~m}$ en el caso de los medianos y cada $200 \mathrm{~m}$ en el caso de los grandes. Los perímetros y el área superficial de los mantos fueron calculados con el programa ArcView 3.2 (ESRI 2008). 
apex to obtain average length in the 3 zones. Length is directly related to biomass, as described by Rivera and Scrosati (2006).

\section{Surface canopy, biomass, and standing crop}

Sargassum beds were classified as small (1 to $500 \mathrm{~m})$, medium (501 to $1,500 \mathrm{~m})$, or large $(>1,501 \mathrm{~m})$ according to their length (Hernández-Carmona et al. 1990, Casas-Valdez et al. 1993). Sargassum beds run parallel to the shoreline. Bed length is the linear distance of each bed parallel to the shore, and width is the linear distance from near-shore to faroffshore, perpendicular to the shoreline. To avoid exceeding $10 \%$ error, the width of the beds was measured every $50 \mathrm{~m}$ for small beds, every $100 \mathrm{~m}$ for medium beds, and every $200 \mathrm{~m}$ for large beds. Bed perimeters and surface canopy areas were calculated using the ArcView 3.2 software (ESRI 2008).

To determine average algal biomass, quadrats were used as sampling units. All Sargassum thalli in each quadrat were collected by hand and weighed (wet weight [WW]) to the nearest $0.1 \mathrm{~kg}$. Ten quadrats were used for small beds, distributed randomly in the total bed area using a random number chart; 15 quadrats were used for medium beds and 20 quadrats for large beds (Hernández-Carmona et al. 1990, CasasValdez et al. 1993). The standing crop of each Sargassum bed was determined by multiplying its average biomass by its total surface canopy area. The standing crop of each zone was calculated by adding the standing crop of beds in each zone.

For all beds, substratum material (sand, rock, or pebbles), and depth were described for every station. From these observations, an algal bed typology was created. Sea surface temperature was obtained from satellite data for 1988 through 2014 (http://oceanwatch.pifsc.noaa.gov/). The relationships between Sargassum biomass and latitude, and seawater temperature were determined by linear correlation analysis (Cohen et al. 2003).

\section{Spatial and temporal changes}

ANOVA tests determined significant differences in the average biomass, surface canopy, standing crop, and length of the Sargassum species among the 3 zones. Differences among means were analyzed with Tukey's test, with confidence set at $P<0.05$, using SAS 6.12 software. Surface canopy area, biomass, and standing crop of Sargassum beds obtained in spring of 2006 were compared with the estimated data for spring of 1988 (Hernández-Carmona et al. 1990), 1991 (Casas-Valdez et al. 1993), and 1995 (Pacheco-Ruíz et al. 1998). The area surveyed by Pacheco-Ruíz et al. (1998) extended from $26^{\circ} 55^{\prime} \mathrm{N}, 111^{\circ} 58^{\prime} \mathrm{W}$ to $27^{\circ} 57^{\prime} \mathrm{N}, 111^{\circ} 45^{\prime} \mathrm{W}$. The area surveyed by Casas-Valdez et al. (1993) extended from $26^{\circ} 55^{\prime} \mathrm{N}, 111^{\circ} 54^{\prime} \mathrm{W}$ to $26^{\circ} 35^{\prime} \mathrm{N}, 111^{\circ} 45^{\prime} \mathrm{W}$. The area surveyed by Hernández-Carmona et al. (1990) extended from $24^{\circ} 22^{\prime} \mathrm{N}$,
Para determinar la biomasa algal promedio, se usaron los cuadrantes como unidades de muestreo. Todos los talos de Sargassum en cada cuadrante fueron recolectados a mano y pesados (peso húmedo [PH]) al $0.1 \mathrm{~kg}$ más cercano. Se usaron 10 cuadrantes para los mantos pequeños, distribuidos al azar en todo el área superficial, usando una tabla de números aleatorios; se usaron 15 cuadrantes para los mantos medianos y 20 cuadrantes para los mantos grandes (HernándezCarmona et al. 1990, Casas-Valdez et al. 1993). Para determinar la cosecha de cada manto, se multiplicó su biomasa promedio por su área superficial total. Para calcular la cosecha de cada zona, se sumó la cosecha de los mantos en cada zona.

Para todos los mantos, se registraron el tipo de sustrato (arena, roca o canto rodado) y la profundidad de cada estación. A partir de estas observaciones se construyó una tipología del manto algal. La temperatura superficial del mar se obtuvo de datos satelitales para 1988 a 2014 (http:// oceanwatch.pifsc.noaa.gov/). La relación de la biomasa de Sargassum con la latitud y la temperatura del mar se determinó mediante un análisis de correlación lineal (Cohen et al. 2003).

\section{Cambios espaciales y temporales}

Se realizó un análisis de varianza para determinar si existían diferencias significativas entre las 3 zonas en cuanto a la biomasa, área superficial, cosecha y longitud promedio de las especies de Sargassum. Las diferencias entre los valores medios fueron analizadas mediante la prueba de Tukey, con el paquete SAS 6.12, estableciendo un valor de significancia de $P<0.05$. Los datos obtenidos en la primavera de 2006 para el área superficial, la biomasa y la cosecha de los mantos de Sargassum fueron comparados con los datos estimados para la primavera de 1988 (Hernández-Carmona et al. 1990), 1991 (Casas-Valdez et al. 1993) y 1995 (Pacheco-Ruíz et al. 1998). El área de estudio de PachecoRuíz et al. (1998) se extendió de $26^{\circ} 55^{\prime} \mathrm{N}, 111^{\circ} 58^{\prime} \mathrm{W}$ a $27^{\circ} 57^{\prime} \mathrm{N}, 111^{\circ} 45^{\prime} \mathrm{W}$, el de Casas-Valdez et al. (1993) de $26^{\circ} 55^{\prime} \mathrm{N}, 111^{\circ} 54^{\prime} \mathrm{W}$ a $26^{\circ} 35^{\prime} \mathrm{N}, 111^{\circ} 45^{\prime} \mathrm{W}$, y el de HernándezCarmona et al. (1990) de $24^{\circ} 22^{\prime} \mathrm{N}, 110^{\circ} 12^{\prime} \mathrm{W}$ a $24^{\circ} 08^{\prime} \mathrm{N}$, $110^{\circ} 24^{\prime} \mathrm{W}$. Los muestreos se realizaron en primavera ya que Sargassum crece más rápido y alcanza su máxima longitud y biomasa durante esta temporada, como ya se mencionó. Mediante un análisis de varianza se determinó si existían diferencias en la biomasa entre años.

\section{Resultados}

\section{Cambios espaciales}

Se identificaron 2 especies de la familia Sargassaceae: Sargassum sinicola subsp. camouii (EY Dawson) JN Norris \& Yensen y S. lapazeanum Setchell \& NL Gardner. Ambos fueron encontrados en la bahía de La Paz (zona III) con una proporción de talos de $70 \%$ y $30 \%$, respectivamente. En las zonas I y II se encontró solamente $S$. sinicola subsp. camouii. 
$110^{\circ} 12^{\prime} \mathrm{W}$ to $24^{\circ} 08^{\prime} \mathrm{N}, 110^{\circ} 24^{\prime} \mathrm{W}$. Surveys were conducted in spring because Sargassum grows more quickly and reaches its maximum length and biomass during this season, as previously mentioned. An ANOVA test determined if there were significant differences in biomass between the years.

\section{RESULTS}

\section{Spatial changes}

Two species in the family Sargassaceae were identified: Sargassum sinicola subsp. camouii (EY Dawson) JN Norris \& Yensen and S. lapazeanum Setchell \& NL Gardner. Both were found in La Paz Bay (zone III) in a proportion of $70 \%$ and $30 \%$, respectively, of thalli. In zones I and II, only $S$. sinicola subsp. camouii was found.

The shortest $(P=0.00002)$ average thallus length of $S$. sinicola subsp. camouii was recorded at La Paz Bay $(0.9-2.1 \mathrm{~m})$ and the longest $(P=0.00002)$ at Mulegé $(1.5-19.0 \mathrm{~m})$. In zone II, thallus length ranged from 0.1 to $3.0 \mathrm{~m}(n=2,917)$. The length of $S$. lapazeanum in zone III $(0.9-1.3 \mathrm{~m})$ was shorter $(n=1,947 ; P=0.00002)$ than the length of $S$. sinicola subsp. camouii.

Sargassum beds were restricted to shallow waters $(0.30-4.0 \mathrm{~m})$ in zone III, intermediate depths $(0.5-5.0 \mathrm{~m})$ in zone II, and a broad range $(1.0-25 \mathrm{~m})$ in zone I. The holdfasts grow on stone, rocks, and pebbles along exposed and protected shores. Sargassum beds were absent on sandy bottoms.

In 2006, 72 Sargassum beds were located along the southeastern coast of the peninsula. They covered a surface area of $4.22 \mathrm{~km}^{2}$, with an estimated standing crop of 19,206 t. Zone I contained 23 beds, zone II contained 22 beds, and zone III contained 27 beds (Fig. 1, Table 1). The highest average biomass was found at Mulegé (zone I), with significant difference $(P=0.000001)$ with respect to the other 2 zones (Table 1). The greatest surface canopy and standing crop occurred at Concepción Bay (zone II).

In general, the average biomass of Sargassum beds increased with latitude $\left(R^{2}=0.59, P=0.0592\right)$ (Fig. 2), that is, biomass is inversely related to water temperature. Average temperature in spring decreased with increasing latitude (Fig. 3). The average biomass of Sargassum beds decreased with increasing temperature $\left(R^{2}=0.52, P=0.0000\right)$. The length of $S$. sinicola subsp. camouii was correlated with the depth of the beds $\left(R^{2}=0.71, P=0.0000\right)$.

\section{Temporal changes}

Zone I

In 1995, Pacheco-Ruíz et al. (1998) found 3 large beds and the smallest covered an area of $56,000 \mathrm{~m}^{2}$. In 2006, we found 23 smaller beds and surface canopy ranged from 1,450 to $220,000 \mathrm{~m}^{2}$. The surface canopy of Sargassum beds in 1995 was $45 \%$ greater than the surface canopy in 2006
Se registró la menor $(P=0.00002)$ longitud promedio de los talos de $S$. sinicola subsp. camouii en la bahía de La Paz $(0.9-2.1 \mathrm{~m})$ y la mayor $(P=0.00002)$ en Mulegé $(1.5-19.0 \mathrm{~m})$. En la zona II, la longitud fue de 0.1 a $3.0 \mathrm{~m}$ $(n=2,917)$. La longitud de los talos de $S$. lapazeanum en la zona III $(0.9-1.3 \mathrm{~m})$ fue menor $(n=1,947 ; P=0.00002)$ que la de $S$. sinicola subsp. camouii.

Los mantos de Sargassum se encontraron en aguas someras $(0.30-4.0 \mathrm{~m})$ en la zona III, a profundidades intermedias $(0.5-5.0 \mathrm{~m})$ en la zona II y en un amplio intervalo de profundidades $(1.0-25 \mathrm{~m})$ en la zona I. Crecen sobre piedra, rocas y cantos rodados en costas expuestas y protegidas. No se observaron mantos de Sargassum creciendo sobre fondos arenosos.

En 2006, se encontraron 72 mantos de Sargassum en la costa sureste de la península. Cubrieron un área superficial de $4.22 \mathrm{~km}^{2}$, con una cosecha estimada de 19,206 t. Se encontraron 23 mantos en la zona I, 22 en la zona II y 27 en la zona III (Fig. 1, Tabla 1). La biomasa promedio más alta se registró en Mulegé (zona I), observándose diferencias significativas $(P=0.000001)$ con respecto a las otras 2 zonas de estudio (Tabla 1). El área superficial y la cosecha fueron mayores en bahía Concepción (zona II).

En general, la biomasa promedio de los mantos de Sargassum incrementó con la latitud $\left(R^{2}=0.59, P=0.0592\right)$ (Fig. 2), esto es, está inversamente relacionada con la temperatura del agua. La temperatura promedio en primavera decreció a mayor latitud (Fig. 3), mientras que la biomasa promedio de los mantos decreció a mayor temperatura $\left(R^{2}=0.52, P=0.0000\right)$. Se observó una correlación entre la longitud de $S$. sinicola subsp. camouii y la profundidad de los mantos $\left(R^{2}=0.71, P=0.0000\right)$.

\section{Cambios temporales}

\section{Zona I}

En 1995, Pacheco-Ruíz et al. (1998) encontraron 3 mantos grandes y el área superficial del manto más pequeño fue de $56,000 \mathrm{~m}^{2}$. En 2006, encontramos 23 mantos más pequeños y el área superficial varió de 1,450 a 220,000 m². El área superficial de los mantos de Sargassum en 1995 fue 45\% más grande que en 2006 (1.8 y $0.82 \mathrm{~km}^{2}$, respectivamente). La biomasa promedio en $1995\left(5 \mathrm{~kg} \mathrm{~m}^{-2} \mathrm{PH}\right)$ fue $55 \%$ menor que en $2006\left(9.1 \mathrm{~kg} \mathrm{~m}^{-2} \mathrm{PH}\right)$, pero la cosecha en 1995 $(8,256 \mathrm{t} \mathrm{PH})$ fue $18 \%$ mayor que en $2006(6,801 \mathrm{t} \mathrm{PH})$ (Tabla 1).

\section{Zona II}

En 1991, Casas-Valdez et al. (1993) encontraron 25 mantos, mientras que en 2006 encontramos 22. Los mantos de 3 sitios (Buenaventura Norte, Cajede y Tasajera) estuvieron ausentes en 2006. El área superficial de los mantos varió considerablemente entre 1991 y 2006: la superficie de algunos 
Table 1. Temporal changes in the number of beds, surface area, biomass, and standing crop of Sargassum spp. at Mulegé (zone I), Concepción Bay (zone II), and La Paz Bay (zone III).

Tabla 1. Cambios temporales del número de mantos, el área superficial, la biomasa y la cosecha de Sargassum spp. en Mulegé (zona I), Bahía Concepción (zona II) y bahía de La Paz (zona III).

\begin{tabular}{|c|c|c|c|c|c|c|}
\hline \multirow[b]{2}{*}{ Variable } & \multicolumn{2}{|c|}{ Zone I } & \multicolumn{2}{|c|}{ Zone II } & \multicolumn{2}{|c|}{ Zone III } \\
\hline & $1995^{1}$ & 2006 & $1991^{2}$ & 2006 & $1988^{3}$ & 2006 \\
\hline Number of beds & 3 & 23 & 25 & 22 & 34 & 27 \\
\hline Total surface area $\left(\mathrm{km}^{2}\right)$ & 1.8 & 0.82 & 2.1 & 2.3 & 2.6 & 1.1 \\
\hline Minimum surface area $\left(\mathrm{m}^{2}\right)$ & 56,000 & 2,000 & 689 & 1,600 & 689 & 1,170 \\
\hline Maximum surface area $\left(\mathrm{m}^{2}\right)$ & 864,000 & 220,000 & 458,741 & 625,200 & 451,370 & 468,300 \\
\hline Average biomass $\pm \mathrm{SD}\left(\mathrm{kg} \mathrm{m}^{2}\right)$ & $5.0 \pm 0.5$ & $9.1 \pm 2.3$ & 3.4 & $4.1 \pm 1.1$ & 7.3 & $3.1 \pm 1.5$ \\
\hline Minimum biomass $\left(\mathrm{kg} \mathrm{m}^{2}\right)$ & - & 3.4 & 1.9 & 1.5 & 2.3 & 2.1 \\
\hline Maximum biomass $\left(\mathrm{kg} \mathrm{m}^{2}\right)$ & - & 33.4 & 5.6 & 5.8 & 16.6 & 4.8 \\
\hline Standing crop (ton) & 8,256 & 6,801 & 7,250 & 9,584 & 18,901 & 2,821 \\
\hline
\end{tabular}

${ }^{1}$ Pacheco-Ruíz et al. (1998), ${ }^{2}$ Casas-Valdez et al. (1993), ${ }^{3}$ Hernández-Carmona et al. (1990).

(1.8 and $0.82 \mathrm{~km}^{2}$, respectively). While the average biomass reported in $1995\left(5 \mathrm{~kg} \mathrm{~m}^{-2} \mathrm{WW}\right)$ was 55\% lower than in 2006 $\left(9.1 \mathrm{~kg} \mathrm{~m}^{-2} \mathrm{WW}\right)$, the standing crop in $1995(8,256 \mathrm{t} \mathrm{WW})$ was $18 \%$ greater than in $2006(6,801 \mathrm{t} \mathrm{WW})$ (Table 1).

\section{Zone II}

While Casas-Valdez et al. (1993) found 25 beds in 1991, we found 22 beds in 2006. The beds at 3 sites (Buenaventura Norte, Cajede, and Tasajera) were absent in 2006. The surface canopy of beds showed high variations between 1991 and 2006: the area of some remained about the same, such as bed $29\left(27,374\right.$ to $\left.27,200 \mathrm{~m}^{2}\right)$ and bed $24(49,070$ to $\left.46,700 \mathrm{~m}^{2}\right)$; the area of some increased enormously, such as bed $45\left(65,446\right.$ to $\left.625,200 \mathrm{~m}^{2}\right)$, bed $37\left(689\right.$ to $\left.137,750 \mathrm{~m}^{2}\right)$, and bed $25\left(9,609\right.$ to $\left.382,800 \mathrm{~m}^{2}\right)$; and the area of some decreased significantly, such as bed $43(458,741$ to $\left.14,950 \mathrm{~m}^{2}\right)$, bed $39\left(145,847\right.$ to $\left.7,200 \mathrm{~m}^{2}\right)$, and bed 31 $\left(108,062\right.$ to $\left.1,600 \mathrm{~m}^{2}\right)$.

In 1991, biomass varied from $1.9 \mathrm{~kg} \mathrm{~m}^{-2} \mathrm{WW}$ at bed 31 to $5.6 \mathrm{~kg} \mathrm{~m}^{-2} \mathrm{WW}$ at bed 45. In 2006, biomass varied from $1.5 \mathrm{~kg} \mathrm{~m}^{-2} \mathrm{WW}$ at bed 24 to $5.8 \mathrm{~kg} \mathrm{~m}^{-2} \mathrm{WW}$ at bed 27 . Average biomass was significantly higher $(P=0.00244)$ in 2006 $\left(4.1 \mathrm{~kg} \mathrm{~m}^{-2} \mathrm{WW}\right)$ than in 1991 (3.4 $\left.\mathrm{kg} \mathrm{m}^{-2} \mathrm{WW}\right)$. Biomass in 2006 was higher than in 1991 at all beds, except beds 45, 44, and 24. Standing crop in 2006 was 9,584 t WW, 24\% higher than in 1991 (7,250 t WW) (Table 1).

\section{Zone III}

While 34 beds were found in 1988 by HernándezCarmona et al. (1990), in 2006 we found 27 beds. The disappearance of large beds at Ensenada Verde-Puertecitos $\left(451,370 \mathrm{~m}^{2}\right)$, Sur de Las Ánimas $\left(60,653 \mathrm{~m}^{2}\right)$, and Ensenada mantos permaneció casi igual, como la de los mantos 29 $\left(27,374\right.$ a $\left.27,200 \mathrm{~m}^{2}\right)$ y $24\left(49,070\right.$ a $\left.46,700 \mathrm{~m}^{2}\right)$; la superficie de algunos aumentó mucho, como la de los mantos 45 $\left(65,446\right.$ a $\left.625,200 \mathrm{~m}^{2}\right), 37\left(689\right.$ a $\left.137,750 \mathrm{~m}^{2}\right)$ y $25(9,609$ a $\left.382,800 \mathrm{~m}^{2}\right)$; y la superficie de algunos disminuyó mucho, como la de los mantos $43(458,741$ a 14,950 m²), 39 (145,847 a $\left.7,200 \mathrm{~m}^{2}\right)$ y $31\left(108,062\right.$ a $\left.1,600 \mathrm{~m}^{2}\right)$.

En 1991, la biomasa varió de $1.9 \mathrm{~kg} \mathrm{~m}^{-2} \mathrm{PH}$ en el manto 31 a $5.6 \mathrm{~kg} \mathrm{~m}^{-2} \mathrm{PH}$ en el manto 45. En 2006, la biomasa varió de $1.5 \mathrm{~kg} \mathrm{~m}^{-2} \mathrm{PH}$ en el manto 24 a $5.8 \mathrm{~kg} \mathrm{~m}^{-2} \mathrm{PH}$ en el manto 27. La biomasa promedio fue significativamente mayor $(P=0.00244)$ en $2006\left(4.1 \mathrm{~kg} \mathrm{~m}^{-2} \mathrm{PH}\right)$ que en 1991 (3.4 $\left.\mathrm{kg} \mathrm{m}^{-2} \mathrm{PH}\right)$. La biomasa en 2006 fue mayor que en 1991 en todos los mantos, excepto en los mantos 45,44 y 24 . La cosecha fue 24\% mayor en $2006(9,584 \mathrm{t} \mathrm{PH})$ que en 1991 $(7,250$ t PH) (Tabla 1).

\section{Zona III}

En 1988, Hernández-Carmona et al. (1990) encontraron 34 mantos, mientras que en 2006 encontramos 27. La desaparición de mantos grandes en Ensenada Verde-Puertecitos $\left(451,370 \mathrm{~m}^{2}\right)$, Sur de Las Ánimas $\left(60,653 \mathrm{~m}^{2}\right)$ y Ensenada San Gabriel en la isla Espíritu Santo $\left(162,021 \mathrm{~m}^{2}\right)$ es importante. El área superficial total de los mantos de Sargassum en 1988 fue $2.6 \mathrm{~km}^{2}$, más de 2 veces la superficie medida en $2006\left(1.1 \mathrm{~km}^{2}\right)$ (Tabla 1). El área superficial de todos los mantos fue mayor en 1988 que en 2006, excepto el de los mantos $68\left(1988=24,564 \mathrm{~m}^{2} ; 2006=43,500 \mathrm{~m}^{2}\right) \mathrm{y}$ $57\left(1988=7,436 \mathrm{~m}^{2} ; 2006=50,900 \mathrm{~m}^{2}\right)$.

La biomasa promedio en $1988\left(7.3 \mathrm{~kg} \mathrm{~m}^{-2} \mathrm{PH}\right)$ fue significativamente mayor (58\%) que en $2006\left(3.1 \mathrm{~kg} \mathrm{~m}^{-2} \mathrm{PH}\right)$ $(P=0.000001)$. La biomasa para todos los mantos fue menor en 2006 que en 1988 (Tabla 1). Los mantos que presentaron 


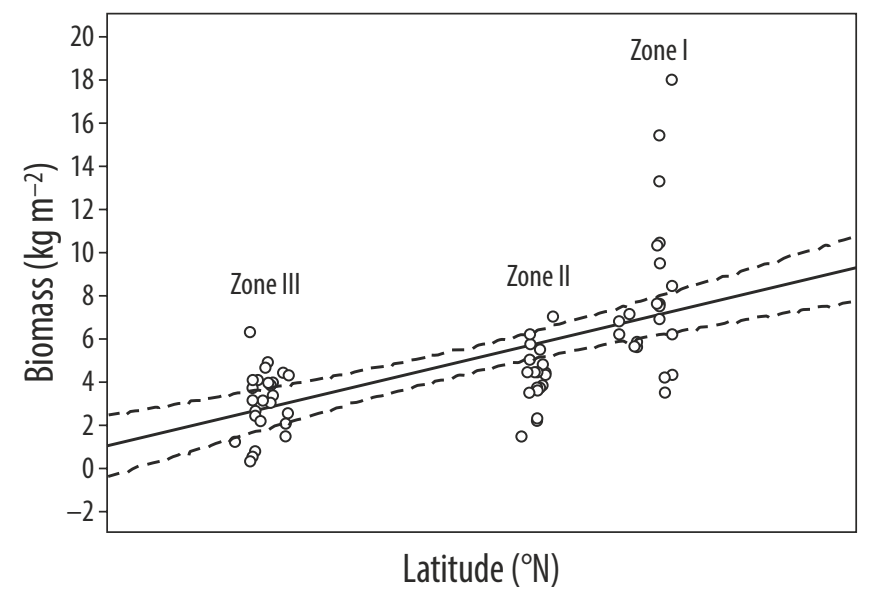

Figure 2. Relationship between Sargassum biomass and latitude along the southeastern coast of the Baja California Peninsula (dashed lines are residuals; solid line is adjusted line).

Figura 2. Relación entre la biomasa de Sargassum y la latitud en la costa sureste de la península de Baja California (las líneas discontinuas son residuales; la línea sólida es la línea ajustada).

San Gabriel at Isla Espíritu Santo $\left(162,021 \mathrm{~m}^{2}\right)$ is important. The total surface of Sargassum beds in 1988 was $2.6 \mathrm{~km}^{2}$, more than twice the area measured in $2006\left(1.1 \mathrm{~km}^{2}\right)$ (Table 1). The surface area of all beds was greater in 1988 than in 2006, except that of beds $68\left(1988=24,564 \mathrm{~m}^{2} ; 2006=\right.$ $\left.43,500 \mathrm{~m}^{2}\right)$ and $57\left(1988=7,436 \mathrm{~m}^{2} ; 2006=50,900 \mathrm{~m}^{2}\right)$.

Average biomass in $1988\left(7.3 \mathrm{~kg} \mathrm{~m}^{-2} \mathrm{WW}\right)$ was significantly greater $(58 \%)$ than in $2006\left(3.1 \mathrm{~kg} \mathrm{~m}^{-2} \mathrm{WW}\right)(P=$ $0.000001)$. Biomass for all beds was lower in 2006 than in 1988 (Table 1). The greatest differences occurred at bed 57 (4.1 $\mathrm{kg} \mathrm{m}^{-2} \mathrm{WW}$ in 2006 and $16.6 \mathrm{~kg} \mathrm{~m}^{-2} \mathrm{WW}$ in 1988, a decline of $61 \%)$ and bed $47\left(3.2 \mathrm{~kg} \mathrm{~m}^{-2} \mathrm{WW}\right.$ in 2006 and $11.2 \mathrm{~kg} \mathrm{~m}^{-2} \mathrm{WW}$ in 1988 , a decline of $71 \%$ ). The standing crop of Sargassum in $2006(2,821 \mathrm{t}$ WW) was $85 \%$ lower than in $1988(18,900 \mathrm{t} \mathrm{WW})$.

\section{DISCUSSION}

\section{Size comparisons}

Sargassum sinicola subsp. camouii was found at all sampling stations, regardless of depth, and was the dominant species in biomass. This is related to its reproductive strategies and persistence over long time periods (Espinoza-Avalos 1990). Sargassum lapazeanum was only abundant in shallow waters with rocky bottoms in zone III.

The highest average length of $S$. sinicola subsp. camouii was recorded in zone I, which results from higher nutrient content in northern waters (Halfar et al. 2004, 2006) and deeper sea floor. Here, $S$. sinicola subsp. camouii occurred at depths from 1 to $25 \mathrm{~m}$. Sargassum gradually increased in average length with distance from the shore (Yeong et al.

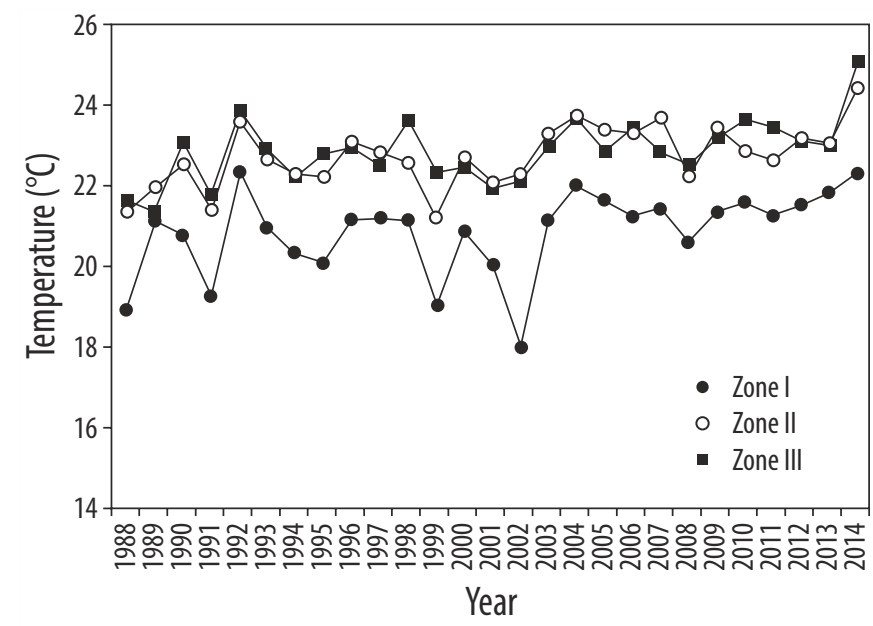

Figure 3. Average sea surface temperature for the 3 study zones: (I) Mulegé, (II) Concepción Bay, and (III) La Paz Bay.

Figura 3. Temperatura superficial del mar promedio para las 3 zonas de estudio: (I) Mulegé, (II) bahía Concepción y (III) bahía de La Paz.

las mayores diferencias fueron el $57\left(4.1 \mathrm{~kg} \mathrm{~m}^{-2} \mathrm{PH}\right.$ en 2006 y $16.6 \mathrm{~kg} \mathrm{~m}^{-2} \mathrm{PH}$ en 1988, una reducción de $61 \%$ ) y el 47 ( $3.2 \mathrm{~kg} \mathrm{~m}^{-2} \mathrm{PH}$ en 2006 y $11.2 \mathrm{~kg} \mathrm{~m}^{-2} \mathrm{PH}$ en 1988 , una reducción de 71\%). La cosecha de Sargassum en 2006 (2,821 t PH) fue $85 \%$ menor que en $1988(18,900 \mathrm{t} \mathrm{PH})$.

\section{DISCUSIÓN}

\section{Comparación de tallas}

Sargassum sinicola subsp. camouii fue encontrada en todas las estaciones de muestreo, independientemente de la profundidad, y fue la especie dominante en biomasa. Esto se puede atribuir a su estrategia de reproducción y su persistencia durante largos periodos de tiempo (Espinoza-Avalos 1990). Sargassum lapazeanum sólo fue abundante en aguas someras con fondos rocosos en la zona III.

La longitud promedio más alta de $S$. sinicola subsp. camouii se registró en la zona I, lo cual se puede atribuir al mayor contenido de nutrientes en aguas del norte (Halfar et al. 2004, 2006) y a la mayor profundidad del fondo marino. Pacheco-Ruíz et al. (1998) registraron una longitud promedio de $1.4 \pm 0.4 \mathrm{~m}$ para esta zona. En nuestro estudio, se observó S. sinicola subsp. camouii a profundidades de 1 a $25 \mathrm{~m}$. La longitud promedio varió de 1.3 a $19 \mathrm{~m}$ (media: $5.2 \pm 3.1 \mathrm{~m}$ ) y aumentó gradualmente a mayor distancia de la costa (Yeong et al. 2012). Ésta es la primera vez que se ha documentado una longitud tan larga en esta zona. Una longitud similar fue registrada para $S$. johnstonii en la costa noreste de la península (Pacheco-Ruíz et al. 1998). La longitud promedio de $S$. sinicola subsp. camouii en las 3 zonas fue mayor que la longitud máxima registrada para la misma especie en bahía 
2012). Pacheco-Ruíz et al. (1998) reported a mean length of $1.4 \pm 0.4 \mathrm{~m}$ for this area. The length of $S$. sinicola subsp. camouii that we recorded ranged from 1.3 to $19 \mathrm{~m}$ (mean: $5.2 \pm 3.1 \mathrm{~m}$ ). Our study is the first to record a long length in this zone. A similar length was reported for S. johnstonii along the northeastern coast of the peninsula (Pacheco-Ruíz et al. 1998). The average length of $S$. sinicola subsp. camouii in the 3 zones was longer than the maximum length reported for the same species in Magdalena Bay $(40 \mathrm{~cm}$; SánchezRodríguez and Cervantes-Duarte 1999).

In Concepción Bay, the average length of $S$. sinicola subsp. camouii was $2.1 \mathrm{~m}$ and maximum length was $7 \mathrm{~m}$ in the spring of 1991 (Casas-Valdez et al. 1993, Núñez-López and Casas-Valdez 1997). In contrast, in 2006, the average length was $0.86 \mathrm{~m}$. In 2006, the beds were located closer to the coast at depths of 0.5 to $5 \mathrm{~m}$; in 1991, the beds were at depths of 0.5 to $15 \mathrm{~m}$ (Casas-Valdez et al. 1993). The depth at which Sargassum grows depends on the presence of hard substrata (Staehr et al. 2008, Tweedley et al. 2008).

The length of $S$. lapazeanum was smaller than that of S. sinicola in Concepción Bay in 1991 (Núñez-López and Casas-Valdez 1997) and at bed 74 in La Paz Bay in 2000 (Rivera and Scrosati 2006). Differences in length are largely determined by genotype characteristics (Núñez-López and Casas-Valdez 1997).

Average Sargassum biomass was lower in zones II and III in 2006 than along the northeastern coast of the peninsula (8.7-22 $\mathrm{kg} \mathrm{m}^{-2} \mathrm{WW}$ in Pacheco-Ruíz et al. 1998), which is a temperate area with lower sea surface temperature and higher nutrient content (Espinoza-Avalos 1993). Zones II and III were similar to other subtropical areas, such as Magdalena Bay $\left(3.8 \mathrm{~kg} \mathrm{~m}^{-2} \mathrm{WW}\right.$; Sánchez-Rodríguez and CervantesDuarte 1999). Zone I in 2006 is consistent with the 2 southern regions ( 8 and 9) studied by Pacheco-Ruíz et al. (1998) in 1995. The average biomass was $9.1 \mathrm{~kg} \mathrm{~m}^{-2} \mathrm{WW}$, higher than the earlier report (5.0 $\mathrm{kg} \mathrm{m}^{-2} \mathrm{WW}$; Pacheco-Ruíz et al. 1998). This is between estimates of subtropical and temperate regions. The biomass in zone I is related to its transitional location between the north and central coastal areas of the peninsula (Espinoza-Avalos 1993). This area is dominated by upwelling currents in the Ballenas Channel, which is colder and more nutrient-rich than the southeastern coast (ÁlvarezBorrego 1983, Álvarez-Molina et al. 2013). According to Yeong et al. (2012), Sargassum species gradually increase in biomass with distance from the shore; in this zone Sargassum thalli can reach $25 \mathrm{~m}$.

Halfar et al. (2004, 2006) measured chlorophyll $a$ as a proxy for nutrients and temperature along the east coast of the peninsula (at $23^{\circ} \mathrm{N}, 25^{\circ} \mathrm{N}, 27^{\circ} \mathrm{N}$, and $29^{\circ} \mathrm{N}$ ) to relate these factors to carbonate production. Chlorophyll $a$ increases northward: $0.3,0.6,1.6$, and $2.2 \mathrm{mg} \mathrm{m}^{-3}$ at $23^{\circ} \mathrm{N}, 25^{\circ} \mathrm{N}, 27^{\circ} \mathrm{N}$, and $29^{\circ} \mathrm{N}$, respectively, which can explain the increase in biomass of Sargassum with increasing latitude.

The standing crop estimated in 2006 (this study) and in 1988 by Hernández-Carmona et al. (1990) and 1991 by
Magdalena (40 cm; Sánchez-Rodríguez y Cervantes-Duarte 1999).

En bahía Concepción, en la primavera de 1991, la longitud promedio de $S$. sinicola subsp. camouii fue de $2.1 \mathrm{~m} \mathrm{y}$ la longitud máxima fue de $7 \mathrm{~m}$ (Casas-Valdez et al. 1993, Núñez-López y Casas-Valdez 1997). En contraste, en 2006, la longitud promedio fue de $0.86 \mathrm{~m}$. En 2006, los mantos se localizaron más cerca de la costa, a profundidades de 0.5 a $5 \mathrm{~m}$; en 1991, los mantos se localizaron a profundidades de 0.5 a 15 m (Casas-Valdez et al. 1993). La profundidad a la cual crece Sargassum depende de la presencia de estrato duro (Staehr et al. 2008, Tweedley et al. 2008).

La longitud de $S$. lapazeanum fue menor que la de S. sinicola en bahía Concepción en 1991 (Núñez-López y Casas-Valdez 1997) y en el manto 74 en la bahía de La Paz en 2000 (Rivera y Scrosati 2006). Las diferencias en longitud son en gran medida determinadas por características genotípicas (Núñez-López y Casas-Valdez 1997).

La biomasa promedio de Sargassum fue menor en las zonas II y III en 2006 que en la costa noreste de la península (8.7-22 $\mathrm{kg} \mathrm{m}^{-2} \mathrm{PH}$ en Pacheco-Ruíz et al. 1998), que es un área templada con menor temperatura superficial del mar y mayor contenido de nutrientes (Espinoza-Avalos 1993). Las zonas II y III fueron similares a otras áreas subtropicales como bahía Magdalena (3.8 kg m² PH; Sánchez-Rodríguez y Cervantes-Duarte 1999). La zona I en 2006 es consistente con 2 áreas sureñas ( 8 y 9) estudiadas por Pacheco-Ruíz et al. (1998) en 1995. La biomasa promedio fue de $9.1 \mathrm{~kg} \mathrm{~m}^{-2} \mathrm{PH}$, mayor que la estimación anterior $\left(5.0 \mathrm{~kg} \mathrm{~m}^{-2} \mathrm{PH}\right.$; PachecoRuíz et al. 1998). Esto es entre estimaciones de regiones subtropicales y templadas. La biomasa en la zona I se relaciona con su ubicación transicional entre las zonas costeras de las partes norte y central de la península (Espinoza-Avalos 1993). Esta área es dominada por corrientes de surgencia en el canal de Ballenas, y las aguas son más frías y más ricas en nutrientes que en la costa sureste (Álvarez-Borrego 1983, Álvarez-Molina et al. 2013). Según Yeong et al. (2012), la biomasa de las especies de Sargassum aumenta gradualmente hacia fuera de la costa; en esta zona, los talos de Sargassum pueden alcanzar $25 \mathrm{~m}$.

Halfar et al. $(2004,2006)$ midieron clorofila $a$ como indicador de nutrientes y temperatura en la costa este de la península (a $23^{\circ} \mathrm{N}, 25^{\circ} \mathrm{N}, 27^{\circ} \mathrm{N}$ y $29^{\circ} \mathrm{N}$ ) para relacionar estos factores con la producción de carbonato. La clorofila $a$ aumenta hacia el norte: $0.3,0.6,1.6$ y $2.2 \mathrm{mg} \mathrm{m}^{-3}$ a $23^{\circ} \mathrm{N}$, $25^{\circ} \mathrm{N}, 27^{\circ} \mathrm{N}$ y $29^{\circ} \mathrm{N}$, respectivamente, lo que puede explicar el aumento en biomasa de Sargassum al aumentar la latitud.

La cosecha estimada en 2006 (este estudio) y en 1988 por Hernández-Carmona et al. (1990) y 1991 por Casas-Valdez et al. (1993) fue menor que la cosecha calculada por PachecoRuíz et al. (1998) en la costa noreste de la península, ya que en esta zona localizada más al norte la temperatura del agua es menor y el contenido de nutrientes es mayor (EspinozaAvalos 1993) que más al sur (Halfar et al. 2004, 2006). 
Casas-Valdez et al. (1993) was lower than the standing crop calculated by Pacheco-Ruíz et al. (1998) along the northeastern coast of the peninsula, since the water temperature is cooler and the nutrient content is higher in this more northern area (Espinoza-Avalos 1993) than further south (Halfar et al. 2004, 2006).

\section{Temporal comparisons}

The difference in biomass in zone III between 1988 and 2006 could result from phenological changes in the beds caused by variations in environmental factors or a reduction of hard substrate surface. Further studies might reveal which factors lead to changes in biomass of Sargassum. Differences in standing crop between 1988 and 2006 are likely related to the disappearance of some important large beds in 2006, which yielded the lowest standing crop and lowest average biomass of the study.

Núñez-López and Casas-Valdez (1996) studied the phenological changes of Sargassum in Concepción Bay and they characterized 8 development stages (seedling, juvenile, young A and B, mature, and senescent A-C). They found that there are geographic and seasonal differences in the phenological stages of Sargassum. For instance, in spring 1991, the largest percentage of Sargassum thalli in bed 45 were young A (developing receptacles) and young B (developed receptacles) plants, while bed 32 contained mature thalli and bed 26 mostly contained senescent B stage thalli. During spring 1992, bed 45 mostly contained thalli at the young A and B stages, bed 32 contained thalli at the young A stage, and in bed 26 all thalli were juveniles. These 8 phenological stages are directly related to the quantities of fronds and, therefore, to the biomass of Sargassum (Núñez-López and CasasValdez 1997). Differences in the biomass found in Sargassum beds at Concepción Bay between 1991 and 2006, and among the different beds in each year, can be the result of differences in the development stages of the Sargassum thalli at a specific time.

The main factor that affects the location of Sargassum is rocky substrata (Espinoza-Avalos 1993). We found that bed length was related to the surface area of hard substrata. The largest Sargassum beds occur at locations where a larger area is covered by stones, rocks, and/or pebbles (Casas-Valdez et al. 1993). The size of the beaches and hard substrate varies because they are exposed to seasonal and inter-annual dynamics, the first mainly due to wave energy (more intense in winter) and the second due to tropical storms and hurricanes. In the 3 zones, no significant coastal modifications or urbanization have occurred near the beds.

Our findings indicate that the standing crop of Sargassum in 3 zones had varied in 2006 relative to previous years. This variability must be considered to be able to achieve the sustainable exploitation of this abundant resource, which can be used as alginate source in many products, balanced diets, forage, etc. We recommend periodic monitoring of the beds

\section{Comparaciones temporales}

La diferencia en biomasa en la zona III entre 1988 y 2006 podría ser resultado de los cambios fenológicos en los mantos de Sargassum producidos por variaciones de factores ambientales o una disminución de sustrato duro. Estudios futuros podrían indicar cuales son los factores que producen estos cambios. En 2006 se registró la menor cosecha y biomasa promedio del estudio. Las diferencias en la cosecha entre 1988 y 2006 probablemente están relacionadas con la desaparición, en 2006, de algunos mantos grandes.

Núñez-López y Casas-Valdez (1996) estudiaron los cambios fenológicos de Sargassum en bahía Concepción e identificaron 8 fases de desarrollo (plántula, juvenil, joven A, joven $B$, maduro, senescente $A$, senescente $B$, senescente $C$ ). Encontraron que existen diferencias geográficas y estacionales en las etapas fenológicas de Sargassum. Por ejemplo, en la primavera de 1991, el mayor porcentaje de talos de Sargassum en el manto 45 eran jóvenes A (receptáculos en desarrollo) y B (receptáculos desarrollados), mientras que el manto 32 contenía plantas maduras y el manto 26 contenía principalmente plantas senescentes B. En la primavera de 1992, el manto 45 contenía principalmente plantas jóvenes (A y B), el manto 32 contenía plantas jóvenes (A) y en el manto 26 todas las plantas eran juveniles. Estas 8 fases fenológicas están directamente relacionadas con la cantidad de frondas y, por lo tanto, con la biomasa de Sargassum (NúñezLópez y Casas-Valdez 1997). Las diferencias en la biomasa de los mantos de Sargassum en bahía Concepción entre 1991 y 2006, y entre los diferentes mantos en cada año, puede ser resultado de las diferencias en las fases de desarrollo de los talos de Sargassum en un tiempo específico.

El principal factor que afecta la ubicación de Sargassum es el sustrato rocoso (Espinoza-Avalos 1993). Encontramos que había una relación entre la longitud del manto y la superficie del sustrato duro. Los mantos más grandes se encuentran en sitios donde una mayor área está cubierta por piedras, rocas y/o cantos rodados (Casas-Valdez et al. 1993). El tamaño de las playas y los sustratos duros varía porque están expuestos a una dinámica estacional e interanual, las primeras principalmente debido a la energía del oleaje (más intenso en invierno) y los segundos debido a tormentas tropicales y huracanes. En las 3 zonas, no se han observado modificaciones costeras importantes o urbanización cerca de los mantos.

Nuestros resultados muestran que la cosecha de Sargassum en las 3 zonas había variado en 2006 con respecto a los años anteriores. Es importante tomar esta variación en cuenta si se quiere lograr una explotación sustentable de este recurso abundante, el cual puede ser usado como fuente de alginato en muchos productos, dietas balanceadas, forraje, etc. Recomendamos que se realice un monitoreo periódico de los mantos para determinar si el recurso es sustentable. La alta variación del área superficial, la biomasa y la cosecha de los mantos de Sargassum no se debe a actividades 
to determine whether the resource is sustainable. The high variability in surface canopy, biomass, and standing crop of Sargassum beds is not related to anthropogenic activities because Sargassum beds have never been harvested along the east coast of the Baja California Peninsula.

\section{ACKNOWLEDGMENTS}

Luis Aguilar Rosas and Raúl Aguilar Rosas ${ }^{\dagger}$ identified the Sargassum species. José Borges Souza, Ciro Arista de la Rosa, Martín Cuevas Higuera, Efraín Flores Montaño, Enrique Calvillo Espinoza, Javier Alvarez Espinoza, Jesús Espinoza Alvarez, and Pablo Simental Sigala conducted important field work. Ira Fogel (CIBNOR) provided extensive editorial services. MCV, ISR, and RNAR are EDI-IPN and COFAA-IPN fellows.

\section{REFERENCES}

Álvarez-Borrego S. 1983. Gulf of California. In: Ketchum BH (ed.), Estuaries and Enclosed Seas. Elsevier, Amsterdam, pp. 427-449.

Álvarez-Molina LL, Álvarez-Borrego S, Lara-Lara JR, Marinone SG. 2013. Annual and semiannual variations of phytoplankton biomass and production in the central Gulf of California estimated from satellite data $=$ Variaciones anual y semianual de la biomasa y producción fitoplanctónica en el golfo de California central estimadas de datos de satélite. Cienc. Mar. 39: 217-230.

Andrefouet S, Zubia M, Payri C. 2004. Mapping and biomass estimation of the invasive brown algae Turbinaria ornata (Turner) J. Agardh and Sargassum mangarevense (Grunow) Setchell on heterogeneous Tahitian coral reefs using 4-meter resolution IKONOS satellite data. Coral Reefs 23: 26-38.

Casas-Valdez MM. 2009. El alga marina Sargassum (Sargassaceae) en el desarrollo regional. In: Urciaga-García JI, Beltrán-Morales LF, Lluch-Belda D (eds.), Recursos Marinos y Servicios Ambientales en el Desarrollo Regional. Centro de Investigaciones Biológicas del Noroeste, Universidad Autónoma de Baja California Sur, Centro Interdisciplinario de Ciencias Marinas-IPN, La Paz, BCS, Mexico, pp. 139-156.

Casas-Valdez M, Hernández-Contreras H, Marín-Álvarez A, Águila-Ramírez RN, Hernández-Guerrero CJ, SánchezRodríguez I, Carrillo-Domínguez S. 2006a. El alga marina Sargassum (Sargassaceae): Una alternativa tropical para la alimentación de ganado caprino. Rev. Biol. Trop. 54: 83-92.

Casas-Valdez M, Portillo-Clark G, Águila-Ramírez RN, RodríguezAstudillo S, Sánchez-Rodríguez I, Carrillo-Domínguez S. 2006b. Effect of the marine algae Sargassum spp. on the productive parameters and cholesterol content of the brown shrimp, Farfantepenaeus californiensis (Holmes, 1990). Rev. Biol. Mar. Oceanogr. 41: 97-105.

Casas-Valdez M, Sánchez-Rodríguez I, Hernández-Carmona G. 1993. Evaluación de Sargassum spp. en la costa oeste de Bahía Concepción, BCS, México. Inv. Mar. CICIMAR 8: 61-69.

Cohen J, Cohen P, West SG, Aiken LS. 2003. Applied Multiple Regression/correlation Analysis for the Behavioral Sciences. 3rd ed. Lawrence Erlbaum Associates, Mahwah, NJ.

Dawson EY. 1944. Marine algae of the Gulf of California. Allan Hancock Pacific Expeditions 3: 189-454.

Espinoza-Avalos J. 1990. Estructura por edades y reproducción de tres poblaciones de Sargassum sinicola (Phaeophyta, Fucales) en la Bahía de La Paz, Golfo de California. Acta Bot. Mex. 11: $1-9$. antropogénicas ya que nunca se han cosechado los mantos en la costa este de la península de Baja California.

\section{Agradecimientos}

Luis Aguilar Rosas y Raúl Aguilar Rosas ${ }^{\dagger}$ identificaron las especies de Sargassum. José Borges Souza, Ciro Arista de la Rosa, Martín Cuevas Higuera, Efraín Flores Montaño, Enrique Calvillo Espinoza, Javier Alvarez Espinoza, Jesús Espinoza Alvarez y Pablo Simental Sigala colaboraron de forma importante en el trabajo de campo. Ira Fogel (CIBNOR) proporcionó servicios editoriales. MCV, ISR y RNAR son becarios de EDI-IPN y COFAA-IPN.

Traducido al español por Christine Harris.

Espinoza-Avalos J. 1993. Macroalgas marinas del Golfo de California. In: Salazar-Vallejo SI, González NE (eds.), Biodiversidad Marina y Costera de México. Comisión Nacional de Biodiversidad y CIQRO, Mexico City, pp. 328-357.

ESRI. 2008. ArcView Software. Geographical Information System. Environmental Systems Research Institute, Redlands, CA.

Halfar J, Godínez-Orta L, Mutti M, Valdez-Holguín JE, Borges JM. 2004. Nutrients and temperature controls on modern carbonate production: An example from the Gulf of California, Mexico. Geology 32: 213-216.

Halfar J, Godínez-Orta L, Mutti M, Valdez-Holguín JE, Borges JM. 2006. Carbonates calibrated against oceanographic parameters along a latitudinal transect in the Gulf of California, Mexico. Sedimentology 53: 1-24.

Hernández-Carmona G, Casas Valdez M, Fajardo León C, SánchezRodríguez I, Rodríguez-Montesinos E. 1990. Evaluación de Sargassum spp. en la Bahía de La Paz, BCS, México. Inv. Mar. CICIMAR 5: 11-18.

Huerta-Díaz MA, De León-Chavira F, Lares ML, Chee-Barragan A, Siqueiros-Valencia A. 2007. Iron, manganese and trace metal concentrations in seaweeds from the central west coast of the Gulf of California. Appl. Geochem. 22: 1380-1392.

Marín-Álvarez A, Casas M, Carrillo S, Hernández H, Monroy A. 2003. Performance of sheep feed rations with Sargassum spp. sea algae. Cuban J. Agric. Sci. 37: 119-123.

Muñeton-Gómez MS, Hernández-Carmona G. 1993. Crecimiento estacional de Sargassum horridum (Setchell \& Gardner), Phaeophyta, en la Bahía de La Paz, B.C.S. México. Inv. Mar. CICIMAR 8: 23-31.

Muñoz-Ochoa M, Murillo-Álvarez JI, Zermeño-Cervantes LA, Martínez-Díaz SF, Riosmena-Rodríguez R. 2010. Screening of extracts of algae from Baja California Sur, Mexico as reversers of the antibiotic resistance of some pathogenic bacteria. Eur. Rev. Med. Pharmacol. Sci. 14(9): 739-747.

Noiraksar T, Sawayama S, Phauk S, Komatsu T. 2014. Mapping Sargassum beds off the coast of Chon Buri Province, Thailand, using ALOS AVNIR-2 satellite imagery. Bot. Mar. 57: 367-377.

Norris JN. 2010. Marine algae of the northern Gulf of California: Chlorophyta and Phaeophyceae. Smithsonian Contributions to Botany, Smithsonian Institution, Washington, DC.

Núñez-López RA, Casas-Valdez M. 1996. Fenología de las especies de Sargassum (Fucales: Sargassaceae) en tres zonas de la Bahía Concepción, B.C.S., México. Rev. Biol. Trop. 44: 455-464. 
Núñez-López RA, Casas-Valdez M. 1997. Variación estacional de la biomasa y talla de Sargassum spp. (Sargassaceae, Phaeophyta) en la Bahía Concepción, B.C.S., México. Hidrobiológica 7: $19-25$.

Pacheco-Ruíz I, Zertuche-González JA, Chee-Barragán A, BlancoBetancourt R. 1998. Distribution and quantification of Sargassum beds along the west coast of the Gulf of California, Mexico. Bot. Mar. 41: 203-208.

Patrón-Prado M, Acosta-Vargas B, Serviere-Zaragoza E, MéndezRodríguez LC. 2010 Copper and cadmium biosorption by dried seaweed Sargassum sinicola in saline waster. Water, Air, Soil Pollut. 210: 197-202.

Rivera M, Scrosati R. 2006 Population dynamics of Sargassum lapazeanum (Fucales, Phaeophyta) from the Gulf of California, Mexico. Phycologia 45: 178-189.

Rodríguez-Montesinos YE, Arvizu-Higuera D, Hernández-Carmona G. 2008 Seasonal variation in length and chemical constituents of Sargassum sinicola Setchell \& Gardner from Bahia de La Paz, Baja California Sur, México. Phycol. Res. 56: 33-38.

Sánchez-Rodríguez I, Cervantes-Duarte R. 1999 Longitud y biomasa de Sargassum sinicola Setchell et Gardner (Phaeophyta) en Bahía Magdalena, BCS, México. Hidrobiológica 9: 117-124.
Setchell WA, Gardner NL. 1924 Expedition of the California Academy of Sciences to the Gulf of California in 1921. The marine algae. Proc. Calif. Acad. Sci. Ser. 4, 12: 695-949.

Staehr PA, Pedersen MF, Thomsen MS, Wernberg T, Krause-Jensen D. 2008. Invasion of Sargassum muticum in Limfjorden (Denmark) and its possible impact on the indigenous macroalgal community. Mar. Ecol. Prog. Ser. 207: 79-88.

Tweedley JR, Jackson EL, Attrill MJ. 2008. Zostera marina seagrass beds enhance the attachment of the invasive alga Sargassum muticum in soft sediments. Mar. Ecol. Prog. Ser. 354: 305-309.

Yabur R, Bashan Y, Hernández-Carmona G. 2007 Alginate from the macroalgae Sargassum sinicola as a novel source for microbial immobilization material in wastewater treatment and plant growth promotion. J. Appl. Phycol. 19: 43-53.

Yeong BML, Wong CL, Tan SG, Zainudin NAI, Yusoh OM. 2012 Three months' monitoring of environmental factors, biomass, length and size classes variation of Sargassum species at Cape Rachado, Port Dickson. In: Pertanika Journal of Tropical Agricultural Science, Universiti Putra Malaysia Press, Serdang, Solangor, pp. 623-630.

Received October 2015, accepted April 2016. 